\title{
El constructo de "límite" y experiencias anómalas en psíquicos
}

\author{
Alejandro Parra \\ Juan Carlos Argibay \\ Instituto de Psicología Paranormal, \\ Buenos Aires, Argentina
}

\begin{abstract}
Recibido: 20 de julio del 2017 / Aprobado: 18 de enero del 2018
doi: 10.26439/persona2018.n021.3019
\end{abstract}

El presente estudio tiene como objetivo investigar las diferencias entre individuos autodenominados psíquicos (o videntes) y un grupo de control (no psíquicos) en la variable de frecuencia de experiencias perceptuales inusuales (percepción anómala) y el constructo psicológico de límite. Los participantes fueron divididos en psíquicos y no psíquicos (individuos que indican no tener ninguna habilidad extrasensorial, pero que han tenido experiencias psíquicas). Los psíquicos $(N=87)$ y un grupo de control $(N=112)$ recibieron la Escala Cardiff de Percepción Anómala, la Escala de Transliminalidad Revisada, el Cuestionario de Limites y una escala para evaluar sus habilidades psíquicas. Todas las hipótesis fueron confirmadas: los psíquicos puntuaron más alto en transliminalidad, percepción anómala y límite "fino”, en comparación con los no psíquicos. Además, se encontró que el mejor predictor para el grupo de psíquicos era la variable de experiencias anómalas ( $\beta=.073 ; p=.03) y$, en segundo lugar, el límite “fino”. Los psíquicos serían aquellos sobre quienes se puede poner a prueba la "hipótesis de continuidad" del límite de Hartmann debido a su familiaridad con su propia capacidad para alterar la conciencia.

psíquicos / habilidades psíquicas / experiencias perceptuales inusuales

Correo electrónico: rapp@fibertel.com.ar 


\section{The construct "limit" and anomalous experiences in psychics}

This study aims at distinguishing self-named psychics (or clairvoyant) from a control group (non-psychics) considering the variable "frequency of unusual perceptual experiences" (anomalous perception), and the psychological construct "limit”. Participants were divided into psychics and non-psychics (people who had psychic experiences but no extrasensory capacities). Both psychics $(N=87)$ and the control group $(N=112)$ completed the Cardiff Anomalous Perceptions Scale (CAPS), the Revised Transliminality Scale (RTS), the Limits Questionnaire, and a scale to measure their psychic abilities. All the hypotheses were confirmed: psychics had higher scores on transliminality, anomalous perception and "fine limit" in comparison with non-psychics. In addition, the variable "anomalous experiences" was the best predictor for the psychics group $(\beta=.073 ; p=.03)$, followed by the variable "fine limit". Psychics would be those who were able to pass the "continuity hypothesis" of Hartmann's limit due to their familiarity with their own capacity to alter their conscience. 


\section{INTRODUCCIÓN}

El psíquico se refiere a sí mismo, por lo general, como aquel que afirma poseer una o más habilidades paranormales, por ejemplo, clarividencia, precognición, telepatía o, incluso, telequinesis (Anderson, 2006; Krippner, 2010; Schouten, 1994). Un concepto común que los agrupa es sensitivo. Un sensitivo que se autodenomina sanador. Cree que tiene habilidades para la curación de enfermedades o disminuir el dolor a otras personas. Aquellos que utilizan la palabra canalizador consideran que tienen capacidades como médiums y que los espíritus o algún otro tipo de entidad hablan, escriben $u$ operan de alguna forma a través de ellos. Un rabdomante también piensa que tiene la habilidad de encontrar agua $u$ otros objetos o personas extraviadas a través de varillas o péndulos.

Sin embargo, no hay suficientes estudios sobre la personalidad de los sensitivos/ psíquicos como para extraer conclusiones definitivas a fin de producir resultados consistentes para esta justificación teórica. Algunos estudios demuestran que los psíquicos, como grupo, tienden a ser menos ansiosos (puntuaciones bajas de neuroticismo), son más extrovertidos y alcanzan un puntaje alto en responsabilidad (medido con el NEO-PI), en comparación con un grupo de control (no psíquicos, individuos con experiencias, pero sin habilidades); para una revisión, por ejemplo, véase Parra y Villanueva (2011a, 2011b), y Parra (2011a). Otro estudio también halló que suelen tener actitudes más positivas, pensamiento orientado a la acción y óptima capacidad de afrontamiento; piensan de manera que promueven una acción eficaz, aceptan más de los demás y, al mismo tiempo, son más rígidos en su pensamiento respecto a los no psíquicos (Parra, 2011b). Finalmente, otro estudio observó diferencia de género; por ejemplo, los psíquicos varones puntuaban significativamente más alto en disociación, absorción y propensión a la fantasía, en comparación con psíquicas mujeres (Parra y Argibay, 2012).

Un constructo todavía poco explorado en individuos sensitivos o psíquicos es el concepto de límite psicológico, aun cuando el resultado parece bastante obvio. El término límite se emplea para describir el grado en que un sistema está conectado (límite delgado o "fino"), en contraste con separado (límite "grueso"). La metáfora del límite "fino" se puede aplicar en muchos niveles dentro de la jerarquía cognitivo-perceptual y extrapersonal (con las demás personas y el entorno cultural, social, familiar, etcétera). Este concepto ha sido recientemente examinado por Hartmann (1991) y Thalbourne (1999). Sin embargo, la idea de un umbral o límite en la mente no es una idea nueva; Frederic W. H. Myers y William James (Thalbourne, 1999) lo habían explorado hace más de un siglo.

Un estudio de Sherwood y Milner (2004-2005) defiende la idea de que la tendencia a manifestar experiencias psíquicas también podría ser un componente clave en el constructo de límite. La delgadez del límite está asociada a varias experiencias paranormales, y se evidencia en diversos experimentos de percepción extrasensorial (Richards, 1996), en efectos de la intención (PK) sobre organismos biológicos (Palmer, 
Simmonds-Moore y Baumann, 2006), en individuos que practican sanación a distancia o que se consideran reikistas (Krippner, Wickramasekera y Tartz, 2002), y en quienes practican chamanismo (Krippner, Wickramasekera, Wickramasekera y Winstead, 1998). Otras investigaciones no encontraron diferencias entre médiums y un grupo de control (Roxburgh y Roe, 2011), o entre curanderos y un grupo de control (Palmer et al., 2006) en la puntuación en el Boundary Questionnaire. En los médiums, el límite "fino" no correlacionaba con trascendencia espiritual, pero eso sí ocurría en curanderos. Esto sugiere que el límite "fino" tiene una compleja relación con las habilidades psíquicas.

El constructo de transliminalidad se compone de variables tales como absorción, propensión a la fantasía, ideación mágica, creencia paranormal, experiencia mística, hiperestesia (hipersensibilidad a los estímulos del medio ambiente, Thalbourne, 1998, p. 403), creatividad, manía y actitud positiva hacia la interpretación de los sueños. La relación entre la transliminalidad y la experiencia psíquica está implícita en la naturaleza del propio constructo. También se correlaciona con un rango de experiencias y creencias paranormales (Thalbourne, 2009). Algunos estudios han encontrado que la transliminalidad se asocia con resultados en test de percepción extrasensorial (Sanders, Thalbourne y Delin, 2000), mientras que otros han obtenido resultados cercanos a lo esperado por azar (Simmonds, 2003). Houran y Lange (2009) han propuesto que esta relación puede entenderse mejor si se tiene en cuenta las diferencias de género; su análisis de los datos demostró que las mujeres que tenían alta puntuación en transliminalidad y los varones que tenían baja transliminalidad parecían mostrar mayor capacidad extrasensorial.

El límite "fino" se refiere a la relativa conexión de los procesos psicológicos entre sí, es decir, un estilo de pensamiento en "tonos de gris". La variable transliminalidad refleja "la tendencia del material psicológico para atravesar umbrales dentro o fuera de la conciencia" (Thalbourne y Houran, 2000, p. 861). La hipótesis de la transliminalidad sugiere que la fuente inmediata de nuestras percepciones no son nuestros ojos y oídos, sino más bien la conciencia subliminal: las percepciones se procesan primero en un nivel inconsciente, para luego, por lo general, velozmente "cruzar el umbral de la conciencia" (Thalbourne, 1999). El límite "fino" aumenta en aquellos que se consideran a sí mismos psíquicos, o que operan como chamanes o sanadores (Krippner et al., 2002). Respecto a las experiencias anómalas, Thalbourne (1999) señala que "la esquizotipia representa probablemente el concepto empíricamente más cercano a la transliminalidad" (p. 20).

El constructo de frontera psicológica de Hartmann (Hartmann, Harrison y Zborowski, 2001) se refiere a un continuo en la delgadez del límite entre la mente y el cerebro. El vínculo entre el límite "fino" y las experiencias psíquicas no es difícil de conceptualizar; se caracteriza por la empatía, la fluidez del pensamiento, la imaginación y la emoción. Schmeidler (1967) y otros investigadores ya habían notado el grado de "sensibilidad" y "flexibilidad" 
que distingue a los psíquicos (p. 317). En una revisión bibliográfica, Richards (1996) sostiene que a veces las experiencias psi se producen en contextos emocionales e interpersonales dinámicos. Las personas con límites "finos" pueden ser más sensibles a las impresiones psíquicas y desarrollar, en consecuencia, fuertes lazos interpersonales que favorecen a las experiencias interpersonales de psi.

En los últimos años, ha habido un renovado interés por una perspectiva dimensional en la cual las experiencias del espectro psicótico, como las alteraciones del pensamiento y la comunicación, están distribuidas en un continuo en la población general (Carpenter, 2014; Linscott y Van Os, 2010). El origen multifactorial, que abarca desde alteraciones genéticas a factores de riesgo ambiental, podría modular la aparición de diferentes manifestaciones clínicas desde la normalidad a la psicosis clínica (Johns et al., 2004; Parra, 2014). Algunas escalas tienen por objeto evaluar la tendencia a la psicosis, mientras que otras se centran en aspectos particulares del continuo (como delirios o alucinaciones), e influencias de los límites de los síntomas en el ámbito psiquiátrico (López-Ibor, Ortiz y López-Ibor, 2011).

Muchas de las medidas no se limitan a la evaluación exclusiva de la presencia de las percepciones anómalas, sino que, más bien, las evaluaciones se mezclan con alteraciones de otras funciones psíquicas, como, por ejemplo, la Escala de Alucinaciones del Launay-Slade ("Oigo sonidos en mis sueños que parecen tan reales que a veces pienso que existen”). Bell,
Halligan y Ellis (2006) diseñaron la Escala Cardiff de Percepción Anómala (CAPS, por sus siglas en inglés) para medir anomalías perceptivas, pero que no dependen de un contexto psiquiátrico clínico; en cambio, considera a las experiencias subjetivas en un rango de diferentes perspectivas de insight (por ejemplo, el reconocimiento de que el percepto "no está realmente allí, el percepto parece extraño o inusual, o el percepto no es una experiencia sensorial compartida). Por otra parte, el CAPS contiene ítems relacionados con la distorsión de la intensidad perceptual, experiencias en todas las modalidades sensoriales apropiadas y experiencias sensoriales asociadas tradicionalmente con alteraciones en el lóbulo temporal.

\section{Predicciones}

El objetivo de este estudio es comparar un grupo de psíquicos con un grupo de control (con experiencias psíquicas, pero sin habilidades psíquicas) en la misma línea de estudios previos (Parra y Argibay, 2007, 2009, 2013a, 2013b) que examinaron a los psíquicos bajo condiciones controladas. El propósito específico de este estudio es investigar una serie de características psicológicas en psíquicos, tales como alucinaciones y percepciones anómalas (medidas con el CAPS) y el constructo de límite (medido con la Escala de Transliminalidad de Thalbourne y la Escala de Límite de Hartmann). Aunque la mayor parte de este trabajo fue concebida como exploratoria, se decidió llevar a cabo un diseño para relevar posibles diferencias psicológicas usando una base de datos previa (Parra y Argibay, 
2013a, 2013b). Algunas predicciones son que (H1) los psíquicos puntuarán más alto en percepción anómala (medido por el CAPS) y que (H2) la transliminalidad y (H3) la puntuación de límite serán más "finas" en los psíquicos que en el grupo de no psíquicos.

\section{Procedimiento de CATEgorización}

Los participantes fueron convocados mediante correo electrónico y un anuncio subido a internet (www.alipsi.com.ar). Usando los valores cargados en la matriz de datos (SPSS), se empleó un procedimiento de categorización para dividir a la muestra en dos grupos: psíquicos y no psíquicos. Para ello, se usó un punto de corte por la mediana $(\mathrm{Mdn} .=8)$ de la puntuación total de la subescala "Habilidades psi" (llamado Index Psi), que resulta de la suma por participante de las respuestas "Nunca" (0), "Una vez" (1) y "Múltiples veces" (2). El producto de las cuatro preguntas sobre habilidades extrasensoriales (por ejemplo, haber tenido sensaciones o impresiones anómalas/ paranormales estando en lugares desconocidos o un conocimiento extrasensorial tocando objetos, o ver el aura) producen un valor final. Este valor genera un índice cuya puntuación mínima va de cero ("Sin habilidad") a una puntuación máxima de 24 ("Haber reportado todas las habilidades psi”, Media $=8.32$; SD = 4.75). Luego es posible dividir el total del Index Psi de toda la muestra $(\mathrm{N}=199)$ en dos grupos: psíquicos (puntuaciones de 9 a 24 en habilidades psi) y no psíquicos (puntuaciones de 0 a 8 en habilidades psi).

\section{Participantes}

El grupo psíquico estuvo conformado por 87 participantes (67 mujeres, o $77 \%$, y 20 varones, o $23 \%$ ), con edades entre 18 a 65 años $($ Media $=40.33 ; \mathrm{DS}=12.08)$. Todos los participantes tenían algún tipo de entrenamiento en meditación u otras técnicas.

El grupo de los no psíquicos estuvo constituido por 112 participantes (81 mujeres, o $72 \%$, y 31 varones, o $28 \%$ ), con edades entre los 17 y 72 años (Media = 46.15; DS = 13.13). El $50 \%$ de los participantes tenía algún tipo de entrenamiento en meditación u otras técnicas.

\section{Diseño y materiales}

\section{Escala Cardiff de Percepción Anómala (CAPS)}

La Escala Cardiff de Percepción Anómala (Bell et al., 2006) es una escala autoadministrable que consta de 32 ítems, diseñada para evaluar anomalías perceptuales, tales como cambios en los niveles de intensidad sensorial, distorsión del mundo externo, desbordamiento sensorial y alucinaciones no clínicas. Se pidió a los participantes que calificaran cada ítem indicando su respuesta: "Sí" (1) o "No" (0). La puntuación alta indica mayor número de anomalías perceptuales, en un rango de 0 (nada) a 32 (lo más alto). La confiabilidad interna del CAPS es buena, con un coeficiente alfa de Cronbach de .87. La fiabilidad test-retest también se ha encontrado aceptable (Bell et al., 2006).

\section{Escala de Transliminalidad (revisada)}

La Escala de Transliminalidad (revisada) presenta 29 ítems en formato dicotómico 
(verdadero/falso) (Thalbourne, 1998). El concepto de transliminalidad se refiere a una "hipersensibilidad"; esto es, una puntuación alta en este aspecto involucra ciertas experiencias paranormales, experiencias místicas, creatividad, experiencia maniaca fugaz, ideación mágica, absorción, propensión a la fantasía, sensibilidad a estímulos sensoriales y actitud positiva hacia la interpretación de los sueños (Houran, Thalbourne y Hartmann, 2003). La escala de transliminalidad se ha administrado a grandes muestras en una variedad de contextos y culturas, de modo que hay correlaciones débiles, moderadas y fuertes. Según Hartmann (1991), la alta puntuación de transliminalidad está fuertemente correlacionada con el límite "fino".

\section{Cuestionario de Límites (BQ)}

El Cuestionario de Límites (BQ, por sus siglas en inglés) es una escala autoadministrable compuesta por 138 ítems, que incluyen diferentes dimensiones de límite (Hartmann, 1989, 1991; Barbuto y Plummer, 1998, 2000). Se divide en 13 categorías: tipo de límite; dormir/vigilia/sueño; experiencias inusuales; pensamientos/sentimientos; niñez/adolescencia; relaciones interpersonales; sensibilidad; exactitud/ precisión; organizaciones; ropa; opiniones acerca de niños, opiniones sobre personas, naciones y grupos; opiniones acerca de la belleza y la verdad; y experiencias paranormales. El formato de respuesta para cada pregunta va desde 0 (nada) a 4 (mucho). Aproximadamente dos tercios de los ítems están redactados de manera que "Totalmente de acuerdo" representa un límite "fino" y "Totalmente en desacuerdo" indica un límite "grueso". El BQ tiene buena fiabilidad test-retest (más de seis meses, $r=.77$ en dos muestras; véase Funkhauser, Würmle, Cornu y Bahro, 2001).

\section{Procedimiento}

Los tres cuestionarios fueron entregados bajo el pseudotítulo de "Cuestionario de Experiencias Psicológicas", y en orden contrabalanceado para evitar sesgos en sus respuestas. Se dio un solo sobre a cada participante, quien recibió información sobre el estudio. Luego se los invitó a completar las tres escalas de forma voluntaria y anónima en una sola sesión.

\section{Resultados}

Se empleó la prueba $U$ de Mann-Whitney, ya que los resultados no se distribuyeron normalmente. La $U$ resultante se transformó en una puntuación $\mathrm{Z}$ para asignar valores de probabilidad. Todas las comparaciones se llevaron a cabo a una cola.

La H1 predice que el grupo de psíquicos puntuaría más alto en transliminalidad que los no psíquicos. Esta hipótesis fue confirmada $\left(\mathrm{p}<.001, \mathrm{E}_{\mathrm{s}}=.57\right) . \mathrm{La} \mathrm{H} 2$ predice que el grupo de psíquicos puntuaría más alto en percepción anómala que los no psíquicos $(\mathrm{p}<.001)$ y la totalidad de sus subescalas, lo que también se confirmó. Los psíquicos puntuaron hacia el límite "fino", en comparación con el grupo de no psíquicos $\left(\mathrm{p}<.001, \mathrm{E}_{\mathrm{s}}=.71\right)$. Cinco $(38 \%)$ de las 13 subescalas, por ejemplo, experiencias inusuales $(\mathrm{p}<.001)$, pensamientos $(\mathrm{p}<.001)$, interpersonal $(\mathrm{p}=.003)$, organizaciones $(\mathrm{p}=.003)$ y experiencias psíquicas $\left(\mathrm{p}<.001, \mathrm{E}_{\mathrm{s}}=.94\right)$, confirmaron la H3 (véase la tabla 1). 
Tabla 1

Comparación entre psíquicos y no psíquicos en percepción anómala, transliminalidad y límite "fino"

\begin{tabular}{|c|c|c|c|c|c|c|c|}
\hline \multirow[b]{2}{*}{ Variables } & \multicolumn{2}{|c|}{$\begin{array}{l}\text { No psíquico } \\
(\mathrm{N}=112)\end{array}$} & \multicolumn{2}{|c|}{$\begin{array}{l}\text { Psíquico } \\
(\mathrm{N}=87)\end{array}$} & \multirow[b]{2}{*}{$\begin{array}{l}Z \\
(D F= \\
198) \\
\end{array}$} & \multirow[b]{2}{*}{$\mathrm{p}$} & \multirow[b]{2}{*}{$E_{S}$} \\
\hline & Media & DS & Media & DS & & & \\
\hline 1. Intensidad sensorial & 1,65 & 1,50 & 2,35 & 1,40 & 3,16 & .002 & .48 \\
\hline $\begin{array}{l}\text { 2. Experiencia sensorial } \\
\text { no compartida }\end{array}$ & 1,56 & 1,36 & 2,45 & 1,29 & 4,28 & $<.001$ & .67 \\
\hline $\begin{array}{l}\text { 3. Experiencia sensorial } \\
\text { inherente no compartida }\end{array}$ & 0,68 & 0,91 & 1,27 & 1,06 & 4,06 & $<.001$ & .59 \\
\hline $\begin{array}{l}\text { 4. Experiencia sensorial } \\
\text { de origen inexplicable }\end{array}$ & 2,34 & 1,50 & 3,37 & 1,52 & 4,60 & $<.001$ & .68 \\
\hline 5. Distorsión corporal & 0,50 & 0,86 & 0,98 & 1,01 & 3,67 & $<.001$ & .51 \\
\hline 6. Alucinaciones verbales & 0,60 & 0,82 & 1,16 & 1,00 & 3,91 & .001 & .61 \\
\hline 7. Desbordamiento sensorial & 0,72 & 0,69 & 1,01 & 0,70 & 2,75 & $<.001$ & .41 \\
\hline $\begin{array}{l}\text { 8. Escuchar pensamientos } \\
\text { exteriorizados }\end{array}$ & 0,31 & 0,52 & 0,52 & 0,59 & 2,66 & .006 & .37 \\
\hline 9. Lóbulo temporal & 1,69 & 1,05 & 2,13 & 1,10 & 2,49 & .008 & .40 \\
\hline Percepción anómala (CAPS) & 9,67 & 6,34 & 14,45 & 6,23 & 4,68 & .012 & .73 \\
\hline 1. Dormir/vigilia/sueño & 13,43 & 7,96 & 15,30 & 7,65 & 1,64 & .100 & .23 \\
\hline 2. Experiencias inusuales & 17,25 & 8,19 & 23,33 & 8,16 & 4,47 & $<.001$ & .74 \\
\hline 3. Pensamientos & 19,35 & 9,63 & 25,01 & 8,79 & 4,02 & $<.001$ & .57 \\
\hline $\begin{array}{l}\text { 4. Niñez/adolescencia/ } \\
\text { adultez }\end{array}$ & 10,28 & 4,02 & 10,75 & 3,58 & 0,90 & .365 & .12 \\
\hline 5. Relaciones interpersonales & 21,05 & 4,06 & 23,17 & 5,08 & 2,96 & .003 & .46 \\
\hline 6. Sensibilidad & 13,20 & 3,02 & 13,77 & 3,63 & 1,38 & .166 & .17 \\
\hline 7. Exactitud/precisión & 17,48 & 4,73 & 18,17 & 5,52 & 0,67 & .503 & .13 \\
\hline 8. Ropa & 30,68 & 6,23 & 32,88 & 6,09 & 1,91 & .055 & .35 \\
\hline 9. Opiniones acerca de niños & 22,13 & 4,54 & 23,17 & 4,91 & 1,70 & .089 & .21 \\
\hline 10. Organizaciones & 20,74 & 4,58 & 22,25 & 4,49 & 2,09 & .037 & .23 \\
\hline $\begin{array}{l}\text { 11. Personas, naciones } \\
\text { y grupos }\end{array}$ & 27,51 & 6,00 & 29,11 & 5,95 & 1,92 & .054 & .26 \\
\hline 12. Belleza y verdad & 15,35 & 3,54 & 14,95 & 3,47 & 0,55 & .455 & .11 \\
\hline 13. Experiencias paranormales & 9,03 & 5,26 & 14,18 & 5,58 & 5,25 & $<.001$ & .94 \\
\hline Límite "fino" (total) & 237,48 & 40,02 & 265,72 & 39,42 & 4,26 & $<.001$ & .71 \\
\hline Transliminalidad & 12,68 & 5,24 & 9,70 & 5,12 & 4,09 & $<.001$ & .57 \\
\hline
\end{tabular}

Como análisis final post hoc, se exploraron correlaciones entre experiencias anómalas con las dos escalas de límite, transliminalidad y cuestionario de límite.
Se encontró que personas que tenían límites más "finos" tendían a obtener mejores resultados en experiencias anómalas (CAPS) (véase la tabla 2). 
Tabla 2

Correlación entre transliminalidad y límite "fino" con experiencias anómalas en psíquicos/no psíquicos y diferencia entre coeficientes de correlación

\begin{tabular}{|c|c|c|c|c|c|c|c|c|}
\hline \multirow[b]{2}{*}{ Ítems de CAPS } & \multicolumn{4}{|c|}{ Transliminalidad } & \multicolumn{4}{|c|}{ Límite "fino" } \\
\hline & $\begin{array}{l}\text { Psíquicos } \\
\text { Rho }\end{array}$ & $\begin{array}{l}\text { Control } \\
\text { Rho }\end{array}$ & z & $\mathrm{p}$ & $\begin{array}{l}\text { Psíquicos } \\
\text { Rho }\end{array}$ & $\begin{array}{l}\text { Control } \\
\text { Rho }\end{array}$ & z & $\mathrm{p}$ \\
\hline 1. Intensidad sensorial & $.46^{* * *}$ & $.49^{* * *}$ & 0,27 & .39 & $.43^{* * *}$ & $.57^{* * *}$ & 1,29 & .09 \\
\hline $\begin{array}{l}\text { 2. Experiencia sensorial } \\
\text { no compartida }\end{array}$ & $.42^{* * *}$ & $.47^{* * \star}$ & 0,43 & .33 & $.32^{* *}$ & $.49^{* * *}$ & 1,41 & .07 \\
\hline $\begin{array}{l}\text { 3. Experiencia sensorial } \\
\text { inherente no compartida }\end{array}$ & $.51^{* \star *}$ & $.37^{* * *}$ & 1,20 & .11 & $.47^{* *}$ & $.45^{\star \star \star}$ & 0,17 & .43 \\
\hline $\begin{array}{l}\text { 4. Experiencia sensorial } \\
\text { de origen inexplicable }\end{array}$ & $.28^{\star *}$ & $.33^{* *}$ & 0,38 & .35 & .18 & $.39^{* \star *}$ & 1,58 & .05 \\
\hline 5. Distorsión corporal & $.38^{* * *}$ & $.30^{* *}$ & 0,62 & .26 & $.36^{* *}$ & $.33^{* *}$ & 0,23 & .40 \\
\hline 6. Alucinaciones verbales & $.25^{\star}$ & $.28^{* *}$ & 0,22 & .41 & $.33^{* *}$ & $.24^{\star}$ & 0,68 & .24 \\
\hline $\begin{array}{l}\text { 7. Desbordamiento } \\
\text { sensorial }\end{array}$ & $.49^{* * *}$ & $.38^{* \star *}$ & 0,94 & .17 & $.51^{* * *}$ & $.28^{* *}$ & 1,89 & .02 \\
\hline $\begin{array}{l}\text { 8. Escuchar pensamientos } \\
\text { exteriorizados }\end{array}$ & $.25^{*}$ & .16 & 0,65 & .25 & .20 & $.20^{*}$ & 0 & .50 \\
\hline 9. Lóbulo temporal & $.45^{\star * \star}$ & $.53^{* * *}$ & 0,73 & .23 & $.48^{* * *}$ & $.59^{* * *}$ & 1,07 & .14 \\
\hline $\begin{array}{l}\text { Percepción anómala } \\
\text { (CAPS) }\end{array}$ & $.59^{* * *}$ & $.56^{\star * *}$ & 0,31 & .37 & $.53^{\star \star \star}$ & $.60^{* *}$ & 0,71 & .23 \\
\hline
\end{tabular}

${ }^{* * *} p<.001 ;{ }^{* *} p=.01 ;{ }^{*} p<.05$

Utilizando un análisis de transformación $\mathrm{Z}$ de Fisher (Sánchez-Bruno y Borges del Rosal, 2005), se evaluó la diferencia entre dos coeficientes de correlación (psíquico/control) en CAPS/Transliminality y CAPS/Límite "fino". No se encontraron diferencias, excepto una leve diferencia en el factor intensidad sensorial. Además, se empleó una regresión logística binaria para evaluar cuál es el mejor predictor para el grupo de psíquicos (método enter). Para la muestra de 199 casos, los resultados del mejor modelo encontraron que experiencias anómalas era el mejor predictor de la diferencia entre los psíquicos y el grupo de control $\left[\beta=.073 ; \mathrm{GL}=1 ; \mathrm{p}=.031 ; \mathrm{r}^{2}=\right.$ .18 ], y en segundo lugar, si se excluye de la regresión a experiencias anómalas, el límite "fino" permanece como el mejor predictor $\left[\beta=.15 ; \mathrm{GL}=1 ; \mathrm{p}=.004 ; \mathrm{r}^{2}=.15\right]$, con una $\beta$ más alta. Esto sugiere que el límite "fino" puede estar por debajo de la diferenciación entre ambos grupos (véase la tabla 2).

\section{DISCUSIÓN}

El objetivo general de este estudio fue comparar un grupo de psíquicos con un grupo 
de control (no psíquicos) para evaluar una serie de diferencias psicológicas entre ambos grupos. Los resultados muestran que los psíquicos tendían a tener más experiencias perceptuales anómalas, tales como oír voces o experiencias de olores (experiencia sensorial no compartida), ver formas, luces o colores, y oír ruidos o sonidos de origen inexplicable. Por ejemplo, Anderson (1988, citado por Irwin, 2009, p. 98) encontró que las experiencias psíquicas, en general, se correlacionaban positivamente con ideación mágica y esquizotipia en médiums. Otros resultados muestran que el grupo de psíquicos propende a tener límite "fino" y reporta experiencias más inusuales que el grupo de control. Es interesante que estos resultados reflejen una condición fronteriza en términos de experiencias subjetivas de estados de conciencia, cognición y emoción, es decir, asociados a formas de pensar e interactuar con el mundo de una manera más hipersensitiva y empática. Puede que los límites más abstractos, como opiniones (sobre la niñez, personas y organizaciones), precisión y belleza, no sean relevantes para la comprensión de las habilidades de los psíquicos, aunque se necesita más trabajo para delinear específicamente qué límites son relevantes para diferentes tipos de habilidad psíquica.

La puntuación global de transliminalidad y límite es mayor en el grupo de psíquicos (Krippner et al., 2002) y en aquellos que operan como chamanes o videntes (Krippner et al., 1998). Los psíquicos serían posibles candidatos para poner a prueba la "hipótesis de la continuidad" de Hartmann, debido a su familiaridad con sus propias alteraciones de la conciencia y al hecho de que muchos de ellos tienden hacia un límite "fino". Esto tiene implicaciones para la habilidad de resolución de problemas, ya que algunas personas pueden resolver situaciones interpersonales en el extremo opuesto del límite “fino", mientras que otras producen soluciones que surgen de sueños, imágenes hipnagógicas e hipnopómpicas, imaginería, fantasía diurna y otras condiciones asociadas al límite "fino".

Gran parte de la investigación reciente debería ser reconsiderada en relación con variables que determinan la forma en que funcionan estas "fronteras" y los factores que moderan la delgadez del límite, en términos de una mejor comprensión de su relación con las habilidades psíquicas y otras experiencias excepcionales. Posiblemente, una línea fructífera de investigación sea la de Persinger y Makarec (1987), que han explorado la relación entre los signos del lóbulo temporal y los fenómenos parapsíquicos. La intensidad sensorial (escuchar sonidos como si fueran mucho más fuertes de lo que normalmente serían) y las experiencias sensoriales no compartidas (por ejemplo, oír voces, percibir olores o ver cosas que otros no pueden) también fueron predominantes en los psíquicos. Estos hallazgos sugieren que las habilidades psíquicas pueden estar relacionadas con procesos cognitivos que implican una fuerte actividad transliminal, en combinación con una esquizotipia perceptual adaptativa que sirve para moldear las experiencias inusuales de los psíquicos, pero que de ningún modo (o rara vez) se transforman en experiencias que implican disfunción perceptual. 
La variable de transliminalidad también refleja la tendencia del material psicológico a traspasar los umbrales entre el yo y el inconsciente, y se compone de habilidades de absorción, propensión a la fantasía, ideación mágica, sistemas de creencia, experiencia mística e hiperestesia, las cuales - juntas o combinadas - pueden ser facilitadoras de psi. Aquellos cuya conciencia subliminal está "en ebullición" son propensos a experimentar imágenes sensoriales fugaces con mayor intensidad que otros (Houran et al., 2003; Sherwood y Milner, 2004-2005), experiencias esquizotípicas (Thalbourne, 1998; Thalbourne, Keogh y Witt, 2005), así como labilidad del lóbulo temporal (Thalbourne, Crawley y Houran, 2003). Además, Simmonds-Moore (2009-2010) encontró una varianza común entre esquizotipia, transliminalidad y labilidad del lóbulo temporal. Los psíquicos mostraron mayor frecuencia de experiencias anómalas, transliminalidad y límite "fino", en la línea conceptual de aquellos estudios que evalúan medidas de propensión a la fantasía como predictoras de los fenómenos psíquicos (Wilson y Barber, 1983). Thalbourne (1999) sugirió que la susceptibilidad a la hipnosis puede jugar un rol en la transliminalidad (véase Healy, 1984), y puede estar relacionada con algunas diferencias fisiológicas en el procesamiento perceptual de las experiencias psíquicas.

Podemos concluir tentativamente que una constelación de factores interrelacionados que componen el constructo de límite "fino" sirve como una predisposición psicológica para el control de las habilidades psíquicas, que distingue a psíquicos de individuos que no lo son. También proporciona potenciales líneas de trabajo que pueden contribuir al diseño de futuros experimentos, tales como los llevados a cabo por nosotros en años recientes (por ejemplo, véase Parra y Argibay, 2007, 2009, 2013a, 2013b).

\section{Referencias}

Anderson, R. (2006). Psychics, Sensitives, and Somnambules: A Biographical Dictionary. Jefferson, NC: McFarland.

Barbuto, J., y Plummer, B. (1998). Mental Boundaries as a New Dimension of Personality: A Comparison of Hartmann's Boundaries in the Mind and Jung's Psychological Types. Journal of Social Behavior and Personality, 13, 421-436.

Barbuto, J., y Plummer, B. (2000). Mental Boundaries and Jung's Psychological Types: A Profile Analysis. Journal of Psychological Type, 54, 17-21.

Bell, V., Halligan, P. W., y Ellis, H. D. (2006). The Cardiff Anomalous Perceptions Scale (CAPS): A New Validated Measure of Anomalous Perceptual Experience. Schizophrenia Bulletin, 32, 366-377. 
Carpenter, W. T. (2014). It Is Time for a New Paradigm for the Study of Psychoses. Revista de Psiquiatría y Salud Mental, 3(1), 1-3.

Funkhauser, A., Würmle, O., Cornu, C., y Bahro, M. (2001). Dream Life and Intrapsychic Boundaries in the Elderly. Dreaming, 11, 83-88.

Hartmann, E. (1989). Boundaries of Dreams, Boundaries of Dreamers: Thin and Thick Boundaries as a New Personality Dimension. Psychiatric Journal of the University of Ottawa, 14, 557-560.

Hartmann, E. (1991). Boundaries in the Mind. Nueva York, NY: Basic Books.

Hartmann, E., Harrison, R., y Zborowski, M. (2001). Boundaries in the Mind: Past Research and Future Directions. North American Journal of Psychology, 3, 347-368.

Healy, J. (1984). The Happy Princess: Psychological Profile of a Psychic. Journal of the Society for Psychical Research, 52, 289296.

Houran, J., y Lange, R. (2009). Searching for an Optimal Level of Transliminality in Relation to Putative Psi. Journal of the Society for Psychical Research, 73, 92-102.

Houran, J., Thalbourne, M. A., y Hartmann, E. (2003). Comparison of Two Alternative Measures of the Boundary Construct. Perceptual and Motor Skills, 96, 311-323.

Irwin, H. (2009). The Psychology of Paranormal Belief: A Research's Handbook. Hartfield: The University of Hertfordshire Press.

Jaén-Moreno, M. J., Moreno-Díaz, M. J., Luque-Luque, R., y Bell, V. (2014). Validation of the Spanish Version of the Cardiff Anomalous Perceptions Scale in the General Population. Actas Españolas de Psiquiatría, 42(1), 1-8.

Johns, L. C., Cannon, M., Singleton, N., Murray, R. M., Farrell, M., y Brugha, T. (2004). Prevalence and Correlates of Self-Reported Psychotic Symptoms in the British Population. British Journal of Psychiatry, 185, 298-305.

Krippner, S. (2010). Debating Psychic Experience: Human Potential or Human Illusion? Santa Bárbara, CA: ABC Clio.

Krippner, S., Wickramasekera, I., y Tartz, R. (2002). Scoring Thick and Scoring Thin: The Boundaries of Psychic Claimants. Journal of Subtle Energy, 11, 43-61.

Krippner, S., Wickramasekera, I., Wickramasekera, J., y Winstead, C. W. (1998). The Ramtha Phenomenon: Psychological, Phenomenological, and Geomagnetic Data. Journal of the American Society for Psychical Research, 92, 1-24.

Linscott, R. J., y Van Os, J. (2010). Systematic Reviews of Categorical versus Continuum Models in Psychosis: Evidence for Discontinuous Subpopulations Underlying a Psychometric Continuum. Implications for DSM-V, DSM-VI, and DSM-VII. Annual Review of Clinical Psychology, 6, 391-419. 
López-Ibor, J. J., Ortiz, T., y López-Ibor, M. I. (2011). Perception, Experience and Body Identity. Actas Españolas de Psiquiatría, 39(13), 3-118.

Palmer, J., Simmonds-Moore, C. A., y Baumann, S. (2006). Geomagnetic Fields and the Relationship between Human Intentionality and the Hemolysis of Red Blood Cells. Journal of Parapsychology, 70.2, 275-301.

Parra, A. (2011a). ¿Qué es sensibilidad psíquica? Buenos Aires: Longseller.

Parra, A. (2011b). Thinking Styles of Psychic Claimants. Australian Journal of Parapsychology, 11, 61-71.

Parra, A. (2014). Alucinaciones: ¿experiencia o trastorno? Buenos Aires: Teseo, colección UAI.

Parra, A., y Argibay, J. C. (2007). Comparing a Free-Response Psychometry Test with a FreeResponse Visual Imagery Test for a Non-Psychic Sample. Journal of the Society for Psychical Research, 71(887), 91-99.

Parra, A., y Argibay, J. C. (2009). "Face-to-Face” Psychic vs. "Remote” Psychic Readings: Comparing Psychics/Non-Psychic Groups Mediatized by "Token-Object" Effect. Australian Journal of Parapsychology, 9, 57-69.

Parra, A., y Argibay, J. C. (2012). Dissociation, Absorption, Fantasy Proneness and SensationSeeking in Psychic Claimants. Journal of the Society for Psychical Research, 76.4(909), 193-203.

Parra, A., y Argibay, J. C. (2013a). Psi and Death of the Person-Target: An Experiment with Highly Emotional Iconic Representations. NeuroQuantology, 4(1), 537-543.

Parra, A., y Argibay, J. C. (2013b). Anomalous Remote Diagnosis: Mental and Motor Psi Impressions under Iconic Representation of the Person-Target. Journal of Parapsychology, 77, 123-130.

Palmer, J. (1996). Individual Differences in Blind Psychic Readings. Proceedings of the PA Convention, 299-316.

Parra, A., y Villanueva, J. C. (2011a). Mirror-Gazing Facility and Psi: Examining Personality Measures. Journal of the Society for Psychical Research, 75(905), 178-190.

Parra, A., y Villanueva, J. C. (2011b). Exploring Different Personality Traits with Psychic Claimants. En M. Kitenis (Ed.), Proceedings of the 54th Annual Convention of the Parapsychological Association (p. 22). Curitiba, Paraná, Brasil.

Persinger, M. A., y Makarec, K. (1987). Temporal Lobe Epileptic Signs and Correlative Behaviors Displayed by Normal Populations. Journal of Genetic Psychology, 114, 179-195.

Richards, D. G. (1996). Boundaries in the Mind and Subjective Interpersonal Psi. Journal of Parapsychology, 60, 227-240. 
Roxburgh, E. C., y Roe, C. A. (2011). A Survey of Dissociation, Boundary Thinness and Psychological Wellbeing in Spiritualist Mental Mediumship. Journal of Parapsychology, 75.3, 279-299.

Sánchez-Bruno, A., y Borges del Rosal, A. (2005). Transformación Z de Fisher para la determinación de intervalos de confianza del coeficiente de correlación de Pearson. Psicothema, 17(1), 148-153.

Sanders, R. E., Thalbourne, M. A., y Delin, P. S. (2000). Transliminality and the Telepathic Transmission of Emotional States: An Exploratory Study. Journal of the American Society for Psychical Research, 94, 1-24.

Schmeidler, G. R. (1967). ESP Breakthrough: Paranormal Effects in Real Life. Journal of the American Society for Psychical Research, 61, 306-325.

Schouten, S. A. (1994). An Overview of Quantitatively Evaluated Studies with Mediums and Psychics. Journal of the American Society for Psychical Research, 88, 221-254.

Sherwood, S. J., y Milner, M. (2004-2005). The Relationship between Transliminality and Boundary Structure Subscales. Imagination, Cognition and Personality, 24(4), 369-378.

Simmonds, C. A. (2003). Investigating Schizotypy as an Anomaly-Prone Personality (tesis doctoral). University College Northampton, Reino Unido.

Simmonds-Moore, C. A. (2009-2010). Sleep Patterns, Personality, and Subjective Anomalous Experiences. Imagination, Cognition, and Personality, 29(1), 71-86.

Storm, L., y Thalbourne, M. A. (1998-1999). The Transliminal Connection between Paranormal Effects and Personality in an Experiment with the I Ching. European Journal of Parapsychology, 14, 100-124.

Thalbourne, M. A. (1998). Transliminality: Further Correlates and a Short Measure. Journal of the American Society for Psychical Research, 92, 402-419.

Thalbourne, M. A. (1999). Transliminality: A Review. International Journal of Parapsychology, 11, 1-34.

Thalbourne, M. A. (2009). Transliminality, Anomalous Belief and Experience, and Hypnotisability. Australian Journal of Clinical and Experimental Hypnosis, 37, 119-130.

Thalbourne, M. A., Crawley, S. E., y Houran, J. (2003). Temporal Lobe Lability in the Highly Transliminal Mind. Personality and Individual Differences, 34, 1965-1974.

Thalbourne, M. A., y Houran, J. (2000). Transliminality, the Mental Experience Inventory and Tolerance of Ambiguity. Personality and Individual Differences, 28, 853-863. 
Thalbourne, M. A., Keogh, E., y Witt, G. (2005). Transliminality and the Oxford-Liverpool Inventory of Feelings and Experiences. Psychological Reports, 96, 579-585.

Wilson, S. C., y Barber, T. X. (1983). The Fantasy-Prone Personality: Implications for Understanding Imagery, Hypnosis, and Parapsychological Phenomena. En A. A. Sheikh (Ed.), Imagery: Current Theory, Research, and Application (pp. 340-382). Nueva York, NY: John Wiley. 
